\title{
ON THE MONOTONICITY PROPERTIES OF ADDITIVE REPRESENTATION FUNCTIONS
}

\author{
Yong-Gao Chen, András Sárközy, Vera T. Sós and Min Tang
}

If $A$ is a set of positive integers, let $R_{1}(n)$ be the number of solutions of $a+a^{\prime}=n, a$; $a^{\prime} \in A$, and let $R_{2}(n)$ and $R_{3}(n)$ denote the number of solutions with the additional restrictions $a<a^{\prime}$, and $a \leqslant a^{\prime}$ respectively. The monotonicity properties of the three functions $R_{1}(n), R_{2}(n)$, and $R_{3}(n)$ are studied and compared.

\section{INTRODUCTION}

Let $\mathbb{N}$ denote the set of positive integers, let $\mathcal{A} \subset \mathbb{N}$ be an infinite set, and put $A(n)=|\{a: a \leqslant n, a \in \mathcal{A}\}|$. For $n=0,1,2, \ldots$, let

$$
R_{1}(n)=R_{1}(\mathcal{A}, n), \quad R_{2}(n)=R_{2}(\mathcal{A}, n), \quad R_{3}(n)=R_{3}(\mathcal{A}, n)
$$

denote the number of solutions of

$$
\begin{array}{lll}
a+a^{\prime}=n, & a, a^{\prime} \in \mathcal{A}, & \\
a+a^{\prime}=n, & a, a^{\prime} \in \mathcal{A}, & a<a^{\prime} \\
a+a^{\prime}=n, & a, a^{\prime} \in \mathcal{A}, & a \leqslant a^{\prime},
\end{array}
$$

respectively.

Erdős, Sárközy and Sós [3, 4] and Balasubramanian [2] studied the monotonicity properties of the functions $R_{1}(n), R_{2}(n)$ and $R_{3}(n)$. Somewhat unexpectedly, it turned out that the monotonicity properties of the three representation functions differ significantly. In particular, Erdös, Sárközy and Sós proved in [3] that $R_{1}(n)$ can be monotonically increasing from a certain point on only in the trivial way:

THEOREM A. The function $R_{1}(n)$ is eventually increasing; that is, there exists an integer $n_{0}$ with

$$
R_{1}(n+1) \geqslant R_{1}(n) \quad \text { for } n \geqslant n_{0}
$$

Received 29th March, 2005

Research supported by the NSF of China Grant 10471064, the SF of AnHui Province Grant 01046103 and the Hungarian National Foundation for Scientific Research Grant T043623, T042750, T038210, T046378.

Copyright Clearance Centre, Inc. Serial-fee code: 0004-9727/05 \$A2.00+0.00. 
if and only if $\mathbb{N} \backslash A$ is finite; that is, there exists an integer $n_{1}$ with

$$
\mathcal{A} \cap\left\{n_{1}, n_{1}+1, n_{1}+2, \ldots\right\}=\left\{n_{1}, n_{1}+1, n_{1}+2, \ldots\right\}
$$

In [3] the following was also proved.

TheOREM B. If $\mathcal{A} \subset \mathbb{N}$ is an infinite set such that

$$
A(n)=o\left(\frac{n}{\log n}\right),
$$

then the function $R_{2}(n)$ cannot be eventually increasing.

In [3] they also claimed the following result:

THEOREM $C$. Let $\mathcal{B}$ be a set of positive integers such that

(i) $B$ is a "Sidon set", that is,

$$
b_{1}+b_{2}=b_{3}+b_{4}, \quad b_{1}, b_{2}, b_{3}, b_{4} \in \mathcal{B}, \quad b_{1} \leqslant b_{2}, b_{3} \leqslant b_{4}
$$

imply that $b_{1}=b_{3}$ and $b_{2}=b_{4}$,

(ii) all the elements of $B$ are even, and

(iii) $b, b^{\prime} \in B$ implies that $\left(b+b^{\prime}\right) / 2 \notin B$.

Then the complement of $B$, that is, the set

$$
\mathcal{A}=\mathbb{N} \backslash \mathcal{B}
$$

is such that the function $R_{2}(n)=R_{2}(A, n)$ is monotonically increasing.

However, this theorem is false in its original form stated above: it is easy to check that the set $\mathcal{B}=\left\{2,2^{2}, \ldots, 2^{n}, \ldots\right\}$ satisfies conditions (i), (ii) and (iii) in the theorem; but defining $\mathcal{A}$ by (2), we have

$$
R_{2}\left(\mathcal{A}, 2^{n}\right)=2^{n-1}-n+1
$$

and

$$
R_{2}\left(\mathcal{A}, 2^{n}+1\right)=2^{n-1}-n
$$

so that

$$
R_{2}\left(\mathcal{A}, 2^{n}\right)>R_{2}\left(\mathcal{A}, 2^{n}+1\right)
$$

and thus $R_{2}(\mathcal{A}, n)$ is not eventually increasing. The error in the theorem is due to the fact that a computational error was made in the last line of (28) in [3] and thus the formula stated there is wrong.

In [4] Erdös, Sárközy and Sós proved:

THEOREM $D$. If $\mathcal{A} \subset \mathbb{N}$ is an infinite set such that

$$
\lim _{n \rightarrow+\infty} \frac{n-A(n)}{\log n}=+\infty
$$


then we have

$$
\underset{N \rightarrow+\infty}{\limsup } \sum_{k=1}^{N}\left(R_{3}(2 k)-R_{3}(2 k+1)\right)=+\infty
$$

It was also shown in [4] that this result is near the best possible:

TheOREM $\mathrm{E}$. There exists an infinite sequence $\mathcal{A} \subset \mathbb{N}$ such that there are $c(>0), n_{0}$ so that

$$
n-A(n)>c \log n \quad\left(\text { for } n>n_{0}\right)
$$

and

$$
\limsup _{N \rightarrow+\infty} \sum_{k=1}^{N}\left(R_{3}(2 k)-R_{3}(2 k+1)\right)<+\infty
$$

Indeed, they proved this by showing that the set

$$
\mathcal{A}=\mathbb{N} \backslash\left\{17,64, \ldots, 4^{2 k}+1,4^{2 k+1}, \ldots\right\}
$$

satisfies (5) and (6).

In [6], Tang and Chen generalised Theorem $D$ and gave a quantitative form of it. As a corollary, we have

THEOREM $F$. If $\mathcal{A} \subset \mathbb{N}$ is an infinite set such that

$$
\limsup _{n \rightarrow+\infty} \frac{n-A(n)}{\log n}=+\infty
$$

then we have

$$
\limsup _{N \rightarrow+\infty} \sum_{k=1}^{N}\left(R_{3}(2 k)-R_{3}(2 k+1)\right)=+\infty .
$$

(9) implies that $R_{3}(2 k)>R_{3}(2 k+1)$ infinitely often, thus it follows from Theorem $F$ that

TheOREM G. If $\mathcal{A} \subset \mathbb{N}$ is an infinite set such that (8) holds, then the function $R_{3}(n)$ cannot be eventually increasing, that is, there is no $n_{0} \in \mathbb{N}$ with

$$
R_{3}(n+1) \geqslant R_{3}(n) \quad \text { for } n \geqslant n_{0} .
$$

Theorem $\mathrm{G}$ with (8) replacing by (3) has also been proved simultaneously and independently by Balasubramanian [2]. However, the following problem has not been solved yet (see [5, Problem 4]). 
Problem 1. Does there exist an infinite set $\mathcal{A} \subset \mathbb{N}$ such that $\mathbb{N} \backslash \mathcal{A}$ is infinite and $R_{3}(n)$ is eventually increasing?

By Theorem $\mathrm{E}$, the set $\mathcal{A}$ in (7) seems to be a good candidate for being a set possessing the properties described in Problem 1, thus one might like to study the monotonicity of $R_{3}(\mathcal{A}, n)$ for this set $\mathcal{A}$. But for this set and $l \geqslant 2$, we have

$$
R_{3}\left(\mathcal{A}, 4^{2 l}+4^{2 l-2}+2\right)=R_{3}\left(\mathcal{A}, 4^{2 l}+4^{2 l-2}+3\right)+1 .
$$

So the function $R_{3}(\mathcal{A}, n)$ cannot be eventually increasing.

Although Theorem $F$ is near the best possible by Theorem $E$, this is not so with Theorem $G$ which is the consequence of Theorem $F$, and perhaps Theorem $G$ could be improved upon. It is even possible that the answer to the question in Problem 1 is negative; that is, $R_{3}(n)$ can be increasing from a certain point on only in the trivial way.

In this paper our goal is twofold. First we shall show that Theorem $\mathrm{C}$ can be corrected by slightly modifying it. The statement of Theorem $\mathrm{C}$ is true if we replace condition (iii) by

(iii) $\quad b, b^{\prime} \in \mathcal{B}$ implies that $\left(b+b^{\prime}\right) \notin \mathcal{B}$.

Indeed, we shall prove slightly more:

THEOREM 1. Let $\mathcal{B} \subset \mathbb{N}$ be an infinite set all whose elements are even, and write $\mathcal{A}=\mathbb{N} \backslash \mathcal{B}$. Then $R_{2}(n)=R_{2}(\mathcal{A}, n)$ is eventually increasing, that is, there exists an integer $n_{0}$ with

$$
R_{2}(n+1) \geqslant R_{2}(n) \quad \text { for } n \geqslant n_{0},
$$

if and only if

(i) $R_{3}(\mathcal{B}, n) \leqslant 1$ for $n \geqslant n_{0}$ and

(ii) $b, b^{\prime} \in \mathcal{B}, b+b^{\prime} \geqslant n_{0}$ imply that $\left(b+b^{\prime}\right) \notin \mathcal{B}$.

We remark that it can be shown easily by the greedy algorithm that there is an infinite set $\mathcal{B} \subset\{2,4,6, \ldots\}$ such that it satisfies (i) and (ii) in Theorem 1 and we have

$$
B(n)=|\mathcal{B} \cap[0, n]| \gg n^{1 / 3}
$$

(and by using a result of Ajtai, Komlós and Szemerédi [1], with a little work this lower bound could be improved to $\left.\gg(n \log n)^{1 / 3}\right)$. Then the complement $\mathcal{A}=\mathbb{N} \backslash \mathcal{B}$ of $\mathcal{B}$ satisfies

$$
A(n)=|\mathcal{A} \cap[0, n]|=n-B(n)<n-c n^{1 / 3} \quad(\text { for large } n) .
$$

Thus by Theorem 1 it follows:

Corollary 1. There is an infinite set $\mathcal{A} \subset \mathbb{N}$ and $c>0, n_{0}, n_{1}$ such that

$$
A(n)<n-c n^{1 / 3} \quad \text { for } n \geqslant n_{0}
$$


and $R_{2}(\mathcal{A}, n)$ is monotonically increasing for $n \geqslant n_{1}$.

We remark that there is a big gap between the lower and upper bounds given for $A(n)$ in (1) and (11). Unfortunately, we have not been able to tighten this gap and, in particular, we have not been able to answer the following question.

Problem 2. Is it true that if $\mathcal{A} \subset \mathbb{N}$ is an infinite set such that $R_{2}(n)$ is monotonically increasing from a certain point on, then we must have

$$
\limsup _{n \rightarrow+\infty} \frac{A(n)}{n}=1
$$

or, perhaps, even

$$
\lim _{n \rightarrow+\infty} \frac{\mathcal{A}(n)}{n}=1 ?
$$

In the second half of this paper we shall prove a further partial result on $R_{3}(n)$ which seems to indicate that, perhaps, the answer to the question in Problem 1 is negative, that is, $R_{3}(n)$ can be monotonically increasing only in the trivial way. We show if $\mathcal{A}$ is infinite and $R_{3}(n)$ is eventually increasing, then writing $\mathcal{B}=\left\{b_{1}<b_{2}<\cdots\right\}=\mathbb{N} \backslash \mathcal{A}$, by Theorem $\mathrm{G}$ there is a $C(=C(B))>1$ so that

$$
b_{n}>C^{n}
$$

for all large $n$. Now we shall show that if the elements of $\mathcal{B}$ grow quickly, then again $R_{3}(n)$ cannot be eventually increasing:

THEOREM 2. Assume that $B=\left\{b_{1}<b_{2}<\ldots\right\} \subset \mathbb{N}$ is an infinite sequence and define $\mathcal{A}$ by $\mathcal{A}=\mathbb{N} \backslash \mathcal{B}$. If

$$
\lim _{n \rightarrow+\infty}\left(b_{n+1}-b_{n}\right)=+\infty,
$$

then the function $R_{3}(n)=R_{3}(\mathcal{A}, n)$ is not eventually increasing; that is, there is no $n_{0}$ with

$$
R_{3}(n+1) \geqslant R_{3}(n) \quad \text { for } n \geqslant n_{0} .
$$

We could prove other similar sufficient criteria. For example, we can prove that if all sufficiently large $b \in \mathcal{B}$ have the same parity, then $R_{3}(n)$ is not eventually increasing. However, we have not been able to settle Problem 1.

The results above reflect a striking and quite unexpected contrast between the monotonicity properties of the three representation functions: while $R_{1}(n)$ can be monotonically increasing only in the trivial way, by Theorem 1 there are many sets $\mathcal{A}$ satisfying (11) so that $R_{2}(n)$ is monotonically increasing. Finally, $R_{3}(n)$ is closer to $R_{1}(n)$, than to $R_{2}(n)$ : either it is monotonically increasing only in the trivial way or if there is a non-trivial $\mathcal{A}$ with this property then it must be such that it can be obtained from $\mathbb{N}$ by dropping only $<c \log n$ integers up to $\mathrm{n}$ (for infinitely many $\mathrm{n}$ ). 


\section{Proof of TheOREM 1}

Write

$$
\begin{gathered}
B(n)=|\{b: b \leqslant n, b \in \mathcal{B}\}|, \\
\eta(i)= \begin{cases}1 & \text { if } i \in \mathcal{B} \\
0 & \text { if } i \notin \mathcal{B}\end{cases}
\end{gathered}
$$

and

$$
\bar{R}(n)=R_{3}(\mathcal{B}, n)=\left|\left\{\left(b, b^{\prime}\right): b, b^{\prime} \in \mathcal{B}, b \leqslant b^{\prime}, b+b^{\prime}=n\right\}\right|
$$

Then

$$
\begin{aligned}
R_{2}(n) & =\left|\left\{\left(a, a^{\prime}\right): a, a^{\prime} \in \mathcal{A}, a<a^{\prime}, a+a^{\prime}=n\right\}\right| \\
& =\sum_{1 \leqslant i<n / 2}(1-\eta(i))(1-\eta(n-i)) \\
& =\sum_{1 \leqslant i<n / 2} 1-|\{i: 1 \leqslant i \leqslant n-1, i \in \mathcal{B}\}|+\left|\left\{\left(b, b^{\prime}\right): b, b^{\prime} \in \mathcal{B}, b \leqslant b^{\prime}, b+b^{\prime}=n\right\}\right| \\
& =\sum_{1 \leqslant i<n / 2} 1-B(n-1)+\bar{R}(n) .
\end{aligned}
$$

Since the elements of $\mathcal{B}$ are even, thus it follows that

$$
R_{2}(2 k)=(k-1)-B(2 k-2)+\bar{R}(2 k)
$$

and

$$
R_{2}(2 k+1)=k-B(2 k)
$$

then

$$
\begin{aligned}
R_{2}(2 k+1)-R_{2}(2 k) & =1-(B(2 k)-B(2 k-2))-\bar{R}(2 k) \\
& =1-\eta(2 k)-\bar{R}(2 k)
\end{aligned}
$$

and

$$
R_{2}(2 k)-R_{2}(2 k-1)=\bar{R}(2 k) .
$$

The latter is always non-negative, thus (10) holds if and only if (14) is non-negative for $2 k \geqslant n_{0}$ :

$$
1-\eta(2 k)-\bar{R}(2 k) \geqslant 0 \quad\left(\text { for } 2 k \geqslant n_{0}\right)
$$

Assume first that $(10)$ holds. Since $\eta(k) \geqslant 0$, it follows from (15) that

$$
\bar{R}(2 k)=R_{3}(\mathcal{B}, 2 k) \leqslant 1 \quad \text { for } 2 k \geqslant n_{0}
$$


The elements of $\mathcal{B}$ are even, thus

$$
R_{3}(B, 2 k+1)=0 \text { for all } k \in \mathbb{N} .
$$

(i) in the theorem follows from (16) and (17). Moreover, if $b, b^{\prime} \in \mathcal{B}$ and $b+b^{\prime} \geqslant n_{0}$, then writing $b+b^{\prime}=2 k$, we have $R_{3}(\mathcal{B}, 2 k)=\bar{R}(2 k) \geqslant 1$, thus it follows from (15) that $\eta(2 k)=\eta\left(b+b^{\prime}\right)=0$ so that $b+b^{\prime} \notin \mathcal{B}$ which proves (ii) in the theorem.

Assume now that (i) and (ii) in the theorem hold. If $2 k \geqslant n_{0}$, then by (i) we have $\bar{R}(2 k)=R_{3}(\mathcal{B}, 2 k) \leqslant 1$ so that $\bar{R}(2 k)=0$ or 1 . If $\bar{R}(2 k)=0$, then by $\eta(2 k) \leqslant 1$ (15) holds trivially. Finally, if $\bar{R}(2 k)=R_{3}(B, 2 k)=1$, then there are $b, b^{\prime} \in \mathcal{B}$ with $b+b^{\prime}=2 k$. By (ii), it follows that $2 k \notin \mathcal{B}$ then $\eta(2 k)=0$ and thus (15) follows. This completes the proof of Theorem 1 .

\section{Proof of ThEOREM 2}

We shall use proof by contradiction: assume that $\mathcal{B} \subset \mathbb{N}$ satisfies (12), however, (13) holds for some $n_{0}$.

Define $B(n), \eta(i)$ and $\bar{R}(n)=R_{3}(\mathcal{B}, n)$ as in the proof of Theorem 1. Then we have

$$
\begin{aligned}
R_{3}(n) & =\sum_{1 \leqslant i \leqslant n / 2}(1-\eta(i))(1-\eta(n-i)) \\
& =\sum_{1 \leqslant i \leqslant n / 2} 1-B(n-1)-\eta(n / 2)+\bar{R}(n)
\end{aligned}
$$

(here we have $\eta(n / 2)=0$ if $n$ is odd). It follows that

$$
R_{3}(2 k)=k-B(2 k-1)-\eta(k)+\bar{R}(2 k)
$$

and

$$
R_{3}(2 k+1)=k-B(2 k)+\bar{R}(2 k+1)
$$

then

$$
\begin{aligned}
R_{3}(2 k+1)-R_{3}(2 k) & =-(B(2 k)-B(2 k-1))+\eta(k)+(\bar{R}(2 k+1)-\bar{R}(2 k)) \\
& =-\eta(2 k)+\eta(k)+\left(R_{3}(\mathcal{B}, 2 k+1)-R_{3}(\mathcal{B}, 2 k)\right) .
\end{aligned}
$$

Clearly we have $R_{3}(\mathcal{B}, 2 k+1)=R_{2}(\mathcal{B}, 2 k+1)$, and $R_{3}(\mathcal{B}, 2 k)-\eta(k)=R_{2}(\mathcal{B}, 2 k)$ (if $k \in \mathcal{B}$, then $b=k, b^{\prime}=k$ is a solution of $b+b^{\prime}=2 k, b, b^{\prime} \in \mathcal{B}, b \leqslant b^{\prime}$ ) thus (18) can be rewritten as

$$
\begin{aligned}
R_{3}(2 k+1)-R_{3}(2 k) & =-\eta(2 k)+\left(R_{2}(\mathcal{B}, 2 k+1)-R_{2}(\mathcal{B}, 2 k)\right) \\
& \leqslant R_{2}(\mathcal{B}, 2 k+1)-R_{2}(\mathcal{B}, 2 k)
\end{aligned}
$$


It follows from (13) and (19) that

$$
\begin{aligned}
0 & \leqslant-\eta(2 k)+\left(R_{2}(\mathcal{B}, 2 k+1)-R_{2}(\mathcal{B}, 2 k)\right) \\
& \leqslant R_{2}(\mathcal{B}, 2 k+1)-R_{2}(\mathcal{B}, 2 k) \text { for } k \geqslant n_{0} / 2 .
\end{aligned}
$$

Write $\mathcal{B}_{0}=\{b: b \in \mathcal{B}, b+1 \notin \mathcal{B}, 2 \mid b\}, \mathcal{B}_{1}=\{b: b \in \mathcal{B}, b+1 \notin \mathcal{B}, 2 \nmid b\}$. For a set $\mathcal{S}$, define $S(m, n)=\{b: m \leqslant b \leqslant n, b \in \mathcal{S}\}$ and $S(n)=S(1, n)$. By (12) we have at least one of $\mathcal{B}_{0}$ and $\mathcal{B}_{1}$ is an infinite set. Write

$$
M= \begin{cases}\max _{b \in \mathcal{B}_{0}} b & \text { if }\left|\mathcal{B}_{0}\right|<\infty \\ \max _{b \in \mathcal{B}_{1}} b & \text { if }\left|\mathcal{B}_{1}\right|<\infty \\ 1 & \text { others. }\end{cases}
$$

By Theorem $\mathrm{G}$, there exists a constant $C=C(\mathcal{A})$ such that

$$
B(n) \leqslant C \log n
$$

for infinitely many positive integers $n$. By the bipartite method, there are infinitely many positive integers $n$ with

$$
|B(n, 2 n)| \leqslant 2 C \text {. }
$$

For such an integer $n$, let $b_{u}$ be the least $b \in B$ with $b \geqslant 2 n$. Then

$$
\left|B\left(\frac{1}{2} b_{u}, b_{u}\right)\right| \leqslant 2 C+1 \text {. }
$$

for large $n$. Thus, there are infinitely many $b_{u} \in \mathcal{B}$ with (21). Let $b_{u}$ be such one with $b_{u}>M+n_{0}$ and $b_{u+1}-b_{u}>1$, and let $i=0$ or 1 with $b_{u} \in \mathcal{B}_{i}$. Let

$$
v=v(u)=\min _{m \geqslant B\left(b_{u}-b_{u-1}\right)}\left\{b_{m}-b_{m-1}\right\}-2
$$

and

$$
\mathcal{B}_{i}(v)=\left\{\bar{b}_{1}<\bar{b}_{2}<\cdots<\bar{b}_{x}\right\}
$$

By the definition of $M$ and (12), we have $\left|\mathcal{B}_{i}(v)\right| \rightarrow \infty$ as $u \rightarrow \infty$. So $x>2 C+1$ for large $u$. Since $u=B\left(b_{u}\right) \geqslant B\left(b_{u}-b_{u-1}\right)$, we have

$$
\bar{b}_{j} \leqslant v<b_{u}-b_{u-1} \leqslant b_{u}
$$

So

$$
R_{2}\left(B, b_{u}+\bar{b}_{j}\right) \geqslant 1 \quad \text { for } j=1,2, \ldots, x \text {. }
$$

Noting that $b_{u}, \bar{b}_{j} \in \mathcal{B}_{i}$, we have $2 \mid b_{u}+\bar{b}_{j}$. By $b_{u}+\bar{b}_{j} \geqslant b_{u}>n_{0}$ and (20), we have

$$
R_{2}\left(B, b_{u}+\bar{b}_{j}+1\right) \geqslant 1 \quad \text { for } j=1,2, \ldots, x .
$$


Let

$$
b_{u}+\bar{b}_{j}+1=b_{s_{j}}+b_{t_{j}}, \quad b_{s_{j}}<b_{t_{j}}, \quad j=1,2, \ldots, x
$$

Then

$$
b_{t_{j}}>\frac{1}{2}\left(b_{s_{j}}+b_{t_{j}}\right)=\frac{1}{2}\left(b_{u}+\bar{b}_{j}+1\right)>\frac{1}{2} b_{u}
$$

and

$$
b_{t_{j}}<b_{u}+\bar{b}_{j}+1 \leqslant b_{u}+v+1<b_{u}+b_{u+1}-b_{u}=b_{u+1} .
$$

So

$$
b_{t_{j}} \in B\left(\frac{1}{2} b_{u}, b_{u}\right)
$$

By (21) and $x>2 C+1$, there exist $1 \leqslant p<q \leqslant x$ with $t_{p}=t_{q}$. Hence, by (22), we have

$$
0<b_{s_{q}}-b_{s_{p}}=\bar{b}_{q}-\bar{b}_{p} \leqslant v .
$$

So

$$
b_{s_{p}+1}-b_{s_{p}} \leqslant v
$$

If $b_{t_{p}}=b_{u}$, then $b_{s_{p}}=\bar{b}_{p}+1$, a contradiction with $\bar{b}_{p} \in \mathcal{B}_{i}$. Thus, $b_{t_{p}}<b_{u}$ and

$$
b_{s_{p}}=b_{u}+\bar{b}_{p}+1-b_{t_{p}}>b_{u}-b_{u-1},
$$

then $s_{p} \geqslant B\left(b_{u}-b_{u-1}\right)$, a contradiction with (23) and the definition of $v$. This completes the proof of Theorem 2.

\section{REFERENCES}

[1] M. Ajtai, J. Komlós and E. Szemerédi, 'A dense infinite Sidon sequence', European J. Combin. 2 (1981), 1-11.

[2] R. Balasubramanian, 'A note on a result of Erdös, Sárközy and Sós', Acto Arith. 49 (1987), 45-53.

[3] P. Erdős, A. Sárközy and V.T. Sós, 'Problems and results on additive properties of general sequences, IV,', in Number Theory, Proceedings, Ootacamund, India, 1984, Lecture Notes in Mathematics 1122 (Springer-Verlag, Berlin, 1985), pp. 85-104.

[4] P. Erdős, A. Sárközy and V.T. Sós, 'Problems and results on additive properties of general sequences, V', Monatsh. Math. 102 (1986), 183-197.

[5] P. Erdős, A. Sárközy and V.T. Sós, 'On additive properties of general sequences', Discrete Math. 136 (1994), 75-99.

[6] M. Tang and Y.G. Chen, 'On additive properties of general sequences', Bull. Austral. Math. Soc. 71 (2005), 479-485. 
Department of Mathematics

Nanjing Normal University

Nanjing 210097

China

Alfréd Rényi Institute of Mathematics
$\quad$ of the Hungarian Academy of Sciences
P.O. Box 127
H-1364 Budapest
Hungary
Department of Algebra and Number Theory Eötvös Loránd University

H-1117 Budapest

Pázmány Péter sétány $1 / \mathrm{c}$

Hungary

Department of Mathematics

Anhui Normal University

Wuhu 241000

China 